\title{
Diversity in asset allocation
}

Received (in revised form): 13th December, 2007

\section{Robert Brown}

is Chairman of the Global Investment Committee at Watson Wyatt, and discusses the use of multiple sources of risk and return to construct more robust portfolios and improve investment efficiency.

\begin{abstract}
Pension funds have historically relied on investment in equities to generate most of their return and therefore equities have also been the main contributor to risk relative to their obligations. This paper identifies the benefits that should follow from including a wider range of asset classes (subject to acceptable levels of valuation) in an investment portfolio and the changes in thinking and practice required to effect this.
\end{abstract}

Pensions (2008) 13, 79-81. doi:10.1057/palgrave.pm.5950064

Keywords: diversity, efficiency, risk, governance, costs

\section{Building portfolios}

Traditionally, pension funds have invested predominantly in two asset classes - equities and bonds. Equities were chosen to provide additional return, while recognising they are risky. Bonds were chosen largely to limit risks relative to liabilities. The average global pension fund is currently (as at end 2006) made up of approximately 60 per cent equities, 26 per cent bonds and around 14 per cent in other assets, mostly property or real estate. In this average fund, risk is typically dominated by the asset allocation decision - policy risk - with the majority arising from equity volatility.

There are, however, an increasing number of other asset classes and strategies that pension funds could invest in to improve diversity. Examples include different types of bonds (high yield debt, emerging market debt, distressed debt, secured loans, etc), global small cap equity, longterm long-only equity, private equity, hedge funds, commodities, PFI/Infrastructure, timber, weather derivatives and catastrophe bonds. Figure 1 shows the returns and risks expected from some of these assets, based on the Watson Wyatt

Correspondence: Robert Brown, Watson Wyatt Limited, Watson House, London Road, Reigate, RH2 9PQ, UK

Tel: +44 01787284387 ;

E-mail: robert.brown@watsonwyatt.com
Global Asset Model assumptions as at 30th September, 2007.

\section{Diversity principles}

We think of diversification as a technical definition involving the reduction of specific (idiosyncratic) risk within an asset class, and is based on assumptions about risk and correlation.

Diversity on the other hand, involves hedging against the possibility that future assumptions about risk and return are not correct (sadly, quite likely despite the significant effort that we and others put into this activity) by maintaining, at all times, a wider range of exposures to different risk return drivers. In effect, this means trying to construct portfolios of assets that are more robust to different (and unknown) economic and financial environments.

\section{Risk return drivers - how we think about the world}

The first support for diversity in asset allocation comes from consideration of the different risk return drivers to which each asset class is exposed, noting that only a relatively small number have 'pure' exposures. Most assets contain a combination of these drivers.

We think of the investment world as containing a range of drivers (see Figure 2). In addition to the equity risk return driver, the four primary ones that pension funds have a natural appetite 


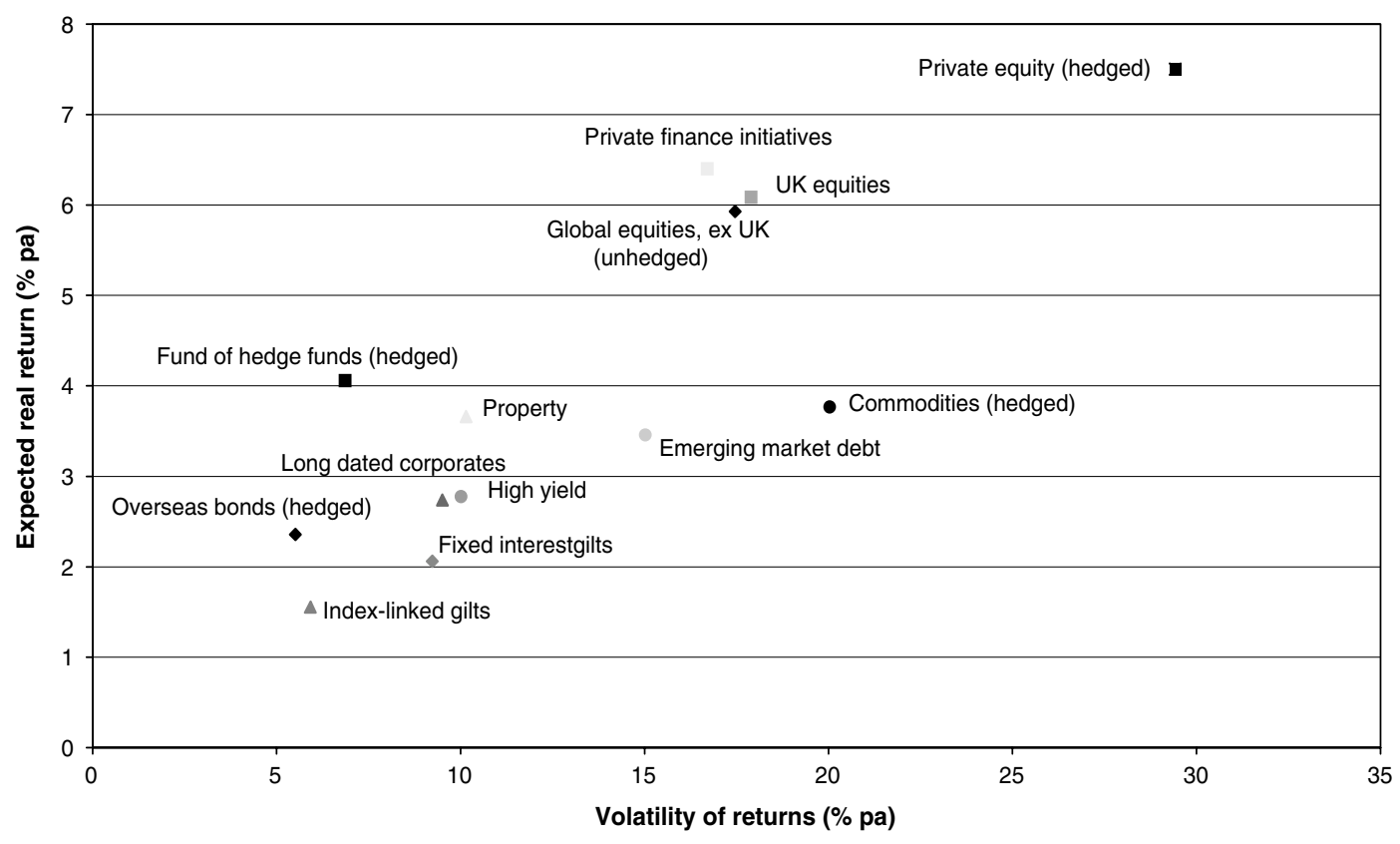

Figure 1: Watson Wyatt Global Asset Model one year risk and return assumptions

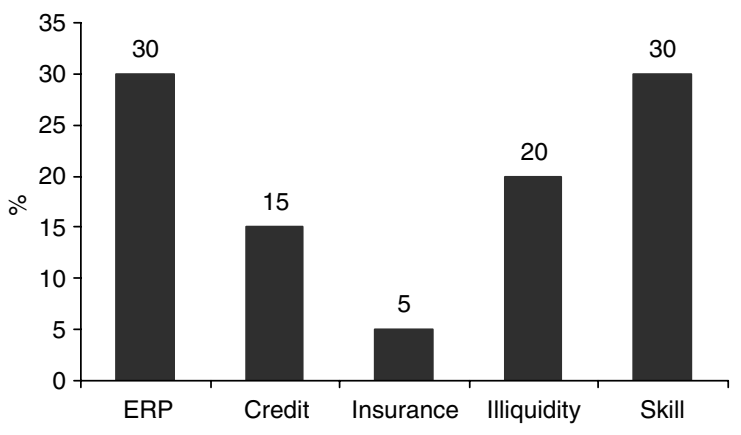

Figure 2: Equilibrium allocations to main risk return drivers

for are:

- Liquidity risk return driver - the additional return investors require to compensate them for an asset's illiquidity.

- Credit risk return driver - the additional return investors in bonds require for taking on the higher level of default risk associated with ratings lower than high quality AAA government grade paper.

- Insurance risk return driver - provision of capital for risk transfer.
- Skill risk return driver - the additional return expected as a result of manager skill.

Considering how these various 'drivers' can be combined, we have created an efficient portfolio that has an optimum blend of long-term exposures to the different risk return drivers and is set according to equilibrium conditions in markets and active management strategies. In the medium term, these portfolio allocations will most likely differ from these long-term targets driven by views on the relative valuations of these premia.

While betas (ie basic market exposures) and alphas (ie added value from active managers) both provide return streams, they each have different characteristics. The excess returns from betas are relatively low compared to their excess risks (ie Sharpe ratios are quite low) are normally relatively correlated, but they tend to be quite reliable - you can be fairly confident that they will be positive over long time horizons.

If you chose wisely, the returns from skill (ie alphas) are attractive and relatively uncorrelated. 
Alphas are, however, hard to come by and returns are unreliable. That is, risk-adjusted net returns are expected to be slightly negative on average. The success of institutional investors in using alphas hinges on the ability to successfully select and balance genuinely skilful managers. The governance requirements for this should not be underestimated.

\section{How much improvement might be possible?}

By including exposures to a wider set of asset classes, a pension fund will gain diversification and improve investment efficiency (as measured by expected outperformance over a proxy for liabilities divided by risk). The extent of this improvement depends on the exact mix chosen and the assumptions adopted. Typically, five per cent exposures to these alternative assets will lead to improvements of the order of two or three per cent. Clearly, combinations of different alternatives can lead to quite meaningful improvements in efficiency.

\section{Pricing and costs}

While in principle, creating more diverse portfolios is a positive aspect in building more efficient portfolios, this has to be subject to reasonable levels of valuation. A sensible investment purchased at the wrong price will most likely end up as a poor investment. On this issue, it is worth noting that up until recently, we had been cautious on a number of alternative asset classes because of compressed risk premia, with a greater emphasis on skill and ERP.

Another important consideration is the potential high costs involved in pursuing this type of strategy which combined with poor manager performance may no longer add up to a value proposition. This is particularly noteworthy in light of the quantum of assets that have flowed into alternative strategies recently, calling into question the ability of some investment managers to continue to deliver good performance and add value once performance fees are included. Indeed, some of our clients have been using the direct route successfully for investing in private equity and hedge funds to reduce fee levels, instead of opting for the fund of funds route which has an associated double layer of fees.

\section{Governance}

Improvements in diversity carry the disadvantage of requiring greater governance. By governance, we mean the combination of resources, expertise and time applied to the management of investment portfolios. In some cases (eg catastrophe bonds) this could considerable. In general, governance requirements tend to be the greatest in the more illiquid, less price transparent, asset classes.

The key governance attributes that we believe are required to be successful with more complex approaches like this are:

- clarity of objectives;

- well-developed and articulated investment beliefs;

- highly developed investment competencies among the principal decision-takers;

- supportive thinking and long-term staying power in the main trustee group;

- effective use of time and resources;

— use of a risk budgeting framework;

- fit for purpose tests applied rigorously to managers.

We observe that excellent investment strategies will tend to produce poor results when they are poorly executed. This suggests that trustees and sponsors should be self-critical when considering their governance competencies against these key attributes before committing heavily to diversity strategies.

\section{In conclusion}

We have suggested that pension funds can benefit from greater diversity in the risk and return drivers embedded in the assets and strategies in their portfolios. The support for this approach is based first on the different risk return drivers that can be assembled in such a portfolio. We note, however, that the principal challenge in this approach lies in the increased governance necessary to be successful and the appropriate valuation levels of these alternatives. A key part of this governance challenge is the imperative to correctly set and manage the attached fees, which can be significant, to ensure maximum benefit for the fund. 